Original

\title{
GLUT-1 immunoexpression in oral epithelial dysplasia, oral squamous cell carcinoma, and verrucous carcinoma
}

\author{
Vidya C. Angadi and Punnya V. Angadi \\ Department of Oral Pathology and Microbiology, KLEVK Institute of Dental Sciences and Hospital, \\ Karnataka, India
}

(Received June 24, 2014; Accepted April 1, 2015)

\begin{abstract}
Glucose transporters, such as GLUT-1, mediate the important mechanisms involved in cellular glucose influx, allowing cells to proliferate and survive. The significance of GLUT-1 expression in oral epithelial dysplasia (OED) and oral squamous cell carcinoma (OSCC) has been less explored, and no study has investigated it in relation to verrucous carcinoma (VC). We evaluated 30 cases each of OED, OSCC, and VC, graded further on the basis of their differentiation, immunohistochemically for GLUT-1 expression, along with 10 specimens of normal oral mucosa (NOM) as controls. In OSCC, GLUT-1 expression increased with the degree of dysplasia and increasing grade $(P<0.001)$. The expression in VC was predominantly membranous and intense, resembling well differentiated OSCC. This increase of GLUT-1 expression in OSCC along with the degree of dysplasia and the histologic grade reflects the expanding glycolytic response to hypoxia. This is the first study to have revealed prominent GLUT-1 expression in $\mathrm{VC}$, highlighting its inherent metabolic capacity. (J Oral Sci 57, 115-122, 2015)
\end{abstract}

Keywords: GLUT-1; oral epithelial dysplasia; oral squamous cell carcinoma; verrucous carcinoma; histologic differentiation; glucose transport.

Correspondence to Dr. Punnya V. Angadi, Department of Oral Pathology and Microbiology, KLEVK Institute of Dental Sciences and Hospital, Belgaum 590010, Karnataka, India

Fax:+91-831-2470640 E-mail: punnya_angadi@rediffmail.com

doi.org/10.2334/josnusd.57.115

DN/JST.JSTAGE/josnusd/57.115

\section{Introduction}

The complex process of oral carcinogenesis is characterized by accumulation of numerous mutations, and genetic and epigenetic aberrations of various genes with diverse roles, particularly the oncogenes and tumor suppressor genes that subsequently serve as the biological hallmarks of cancer (1). Oral squamous cell carcinomas usually follow clinically detectable premalignant lesions including oral leukoplakia, erythroplakia, and oral submucous fibrosis, which histologically represent the process of oral epithelial dysplasia (OED) (2).

OED is the histological manifestation of potentially malignant lesions, for which the rate of progression to oral squamous cell carcinoma (OSCC) reportedly ranges from $6 \%$ to $36 \%(2,3)$. Although OED is diagnosed on the basis of morphological analysis, it is important to recognize that not all cases of severe dysplasia may lead to squamous cell carcinoma, and that paradoxically even mild dysplasia can become malignant, thus emphasizing the fundamental importance of knowledge about the molecular pathophysiology of these lesions (3). Alteration of the normal oral mucosa (NOM) to OED, and ultimately to OSCC, represents an intricate process involving interplay of several pathogenetic factors (4). Accordingly, the molecular events that orchestrate these changes have been a focus of considerable interest (5). One such important molecular event in carcinogenesis that could affect the metabolic capacity of tumor cells is the development of a glycolytic phenotype in response to hypoxia.

GLUT-1 is a glucose transporter that facilitates the transport of glucose into cells, thus helping to regulate energy metabolism, especially in tissues where energy 
supply is inadequate (6). Cancer cells require high energy levels via glycolytic generation of ATP, which enhances their proliferative potential and survival capability. In malignancy-associated cachexia, GLUT-1 overexpession supports increased tumor proliferation at the cost of host tissues. Consequently, GLUT-1 overexpression has been associated with the malignant transformation and progression of several cancers (6).

GLUT-1 is normally expressed in the membranes of red blood cells, brain capillary endothelium, perineurium of peripheral nerves, salivary gland ducts, and the germinal centers of lymph nodes, where it is demonstrable by immunohistochemistry (7).

It has been suggested that altered GLUT- 1 expression is the initial change evident in not only OSCC, but also nonsmall cell lung carcinoma and various other carcinomas including those of the esophagus, colon, colorectum, stomach, breast, uterine cervix and pancreas, being positively correlated with aggressive tumor behavior and poor prognosis (8). Furthermore, enhanced GLUT-1 expression has been noted in premalignant lesions (9).

Although the metabolic consequences of increased glucose transport associated with enhanced GLUT-1 expression are still unclear, its clinical implications in numerous malignancies have been suggested (8). Although GLUT-1 overexpression is consistently evident in patients with head and neck squamous cell carcinoma, its prognostic value has not been analyzed systematically, particularly in the context of OED and OSCC (7). Furthermore, no previous study has investigated this endogenous hypoxic marker in verrucous carcinoma (VC), a less aggressive counterpart of OSCC showing specific clinicopathologic features and molecular changes. The present study was conducted to assess the immunohistochemical expression of GLUT-1 in normal oral mucosa (NOM) in comparison with varying degrees of OED, and different grades of OSCC and VC.

\section{Materials and Methods}

\section{Tissue material}

This study was approved by the KLE VK institute of Dental Sciences, Belgaum ethics committee (3532, June 2012). Paraffin-embedded tissue blocks of previously diagnosed cases of OED (30 cases), OSCC (30 cases), and VC (30 cases) were included. NOM (10 specimens) obtained from gingival and vestibular mucosa during disimpaction of third molars was employed as a control material. The authors re-reviewed the hematoxylin- and eosin-stained slides to confirm the diagnosis for inclusion in the study. The OED cases were classified histopathologically into mild, moderate or severe dysplasia according to the WHO 2005 criteria (10). The OSCC cases were graded according to Broder's criteria into well differentiated squamous cell carcinoma (WDSCC) (10 cases) moderately differentiated squamous cell carcinoma (MDSCC) (10 cases) or poorly differentiated squamous cell carcinoma (PDSCC) (10 cases) (11). These cases of OED, OSCC, and VC along, with NOM, were then subjected to immunohistochemistry for GLUT-1.

\section{Immunohistochemistry}

The super-sensitive one-step polymer-HRP technique (Biogenex Life Sciences, Fremont, CA, USA) was used for immunohistochemical analysis of GLUT-1. Sections 4-5 $\mu \mathrm{m}$ thick were taken from the selected tissue blocks and loaded onto silane-coated slides. Following deparaffinization by heating on a slide warmer and treatment with xylene, the sections were rehydrated in ethanol. They were then placed in a container containing $10 \mathrm{mM}$ sodium citrate buffer ( $\mathrm{pH}$ 6.0) in a commercial microwave antigen retrieval system (EZ-Retriever System, Biogenex Life Sciences) and treated at $96^{\circ} \mathrm{C}$ for two cycles of 12 min each for antigen retrieval. The sections were rinsed in TBS and treated with peroxidase blocking agent for $15 \mathrm{~min}$ and power block for $20 \mathrm{~min}$ to eliminate the endogenous peroxidase and non-specific antigenic sites. Optimally pre-diluted antibody against GLUT-1 (mouse monoclonal: Clone SPM498, Biogenex Life Sciences) was then added to the sections and allowed to stand for $45 \mathrm{~min}$ at room temperature. The sections were then incubated with One-Step Polymer-HRP reagent for 30 min and visualized using freshly prepared diaminobenzidine tetrahydrochloride. The sections were then stained with hematoxylin, dehydrated, cleared, and mounted with DPX. Normal oral mucosa and nerve tissues were employed as positive controls, while endothelial staining of blood vessels, which are known to express GLUT-1, served as the internal control.

\section{Immunohistochemical analysis}

GLUT-1 expression was expressed as the percentage of positivity (score 0 , absence of expression; score 1, 1-25\% of the cells showing positivity; score 2, 25-50\% of the cells positive; score $3,>50 \%$ positive expression), intensity of staining ( 0 , no expression; 1 , mild expression, i.e., less than that of the control; 2 , intense expression similar to that shown by the internal control endothelial cells), and location ( 0 , no staining; 1 , membrane only; 2 , cytoplasmic location; 3 , nuclear expression; 4 , combination of membrane and cytoplasm). Additionally, in OED, the extent of GLUT-1 staining in the epithelium was also evaluated ( 0 , absent; 1 , expression limited to the basal and 


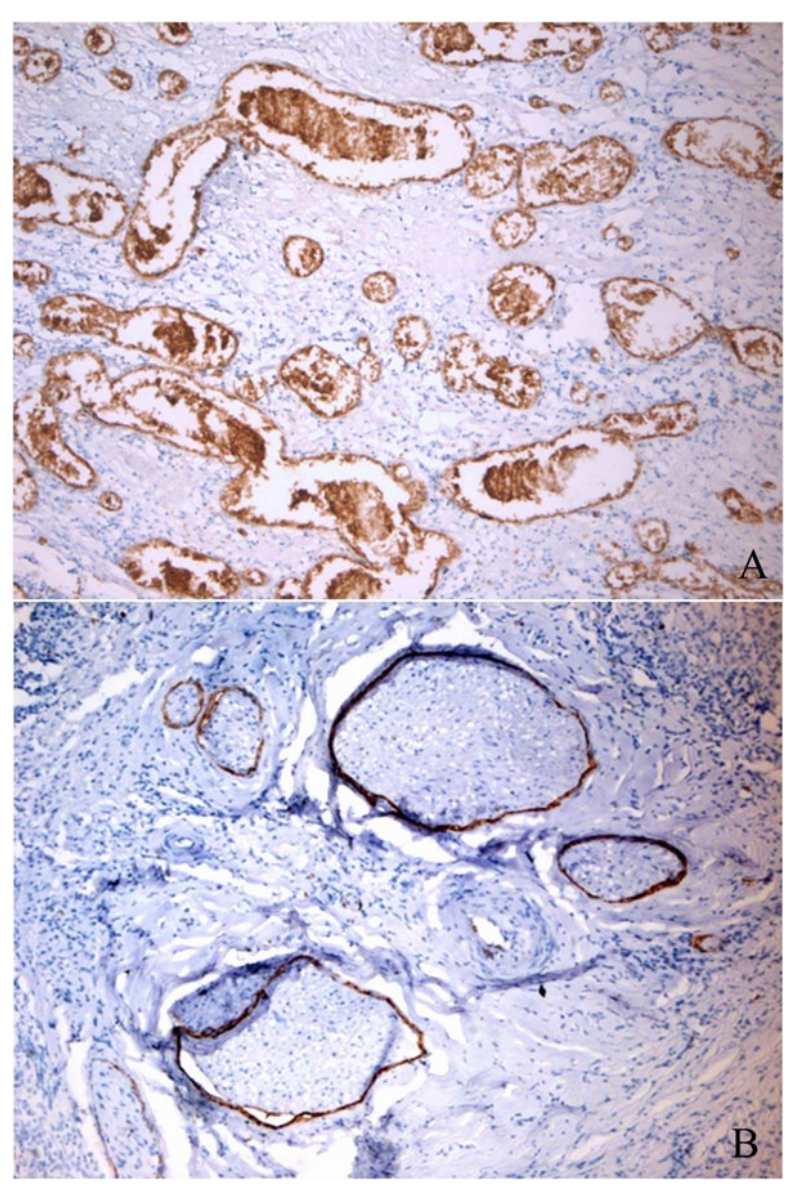

Fig. 1 Intense GLUT-1 expression in the vascular endothelium $(\mathrm{A}, \times 250)$ and perineural tissue $(\mathrm{B}, \times 250)$ used as internal positive controls.

suprabasal layers; 2, expression extending to the spinous layer; 3, extending to the granular layer; 4, extending to the corneal layer). Two independent observers (VA and PA) evaluated all the hematoxylin/eosin- and immunohistochemically stained sections.

\section{Statistical analysis}

GLUT-1 immunoexpression in NOM, OSCC and VC was assessed using chi-square test and Fisher's exact test.

\section{Results}

Erythrocytes, vascular endothelium and perineurium employed as internal positive controls demonstrated strong GLUT-1 positivity. The tumor stroma was negative for GLUT-1 (Fig. 1).

\section{Location}

Most of the cases in the study groups demonstrated predominantly membranous GLUT-1 expression, although two cases of OED, seven cases of $\mathrm{VC}$ and eitht cases of OSCC exhibited combined membranous
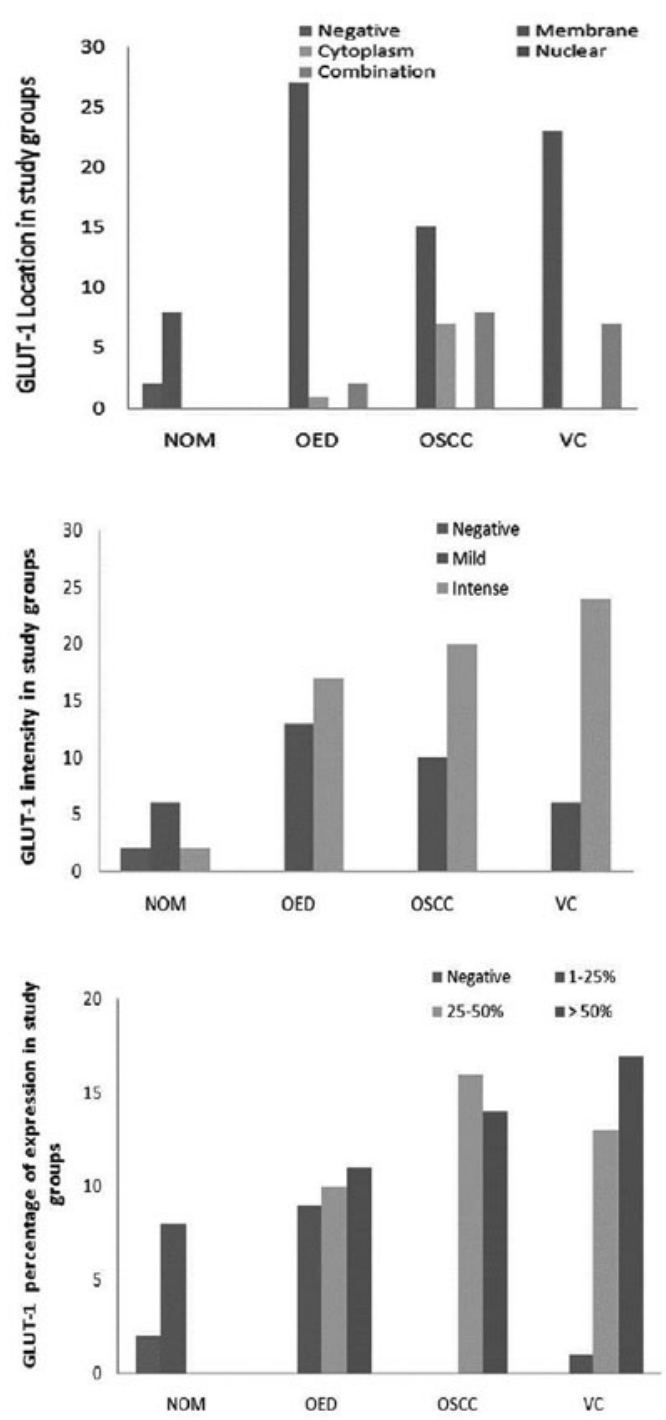

Fig. 2 GLUT-1 immunoexpression, i.e., cellular location, intensity and the percentage of positivity in NOM, OED, OSCC, and VC.

and cytoplasmic expression. This difference in GLUT-1 location was statistically significant $(P<0.001)$ (Fig. 2). Within the OED group, all three grades demonstrated membranous staining predominantly (Figs. 3, 4). The location of GLUT-1 showed a progressive switch from a membranous to a cytoplasmic location, and then to a combination of both, as the grade of OSCC increased $(P=0.030)$ (Figs. 5, 6). Predominantly membranous staining was seen in WDSCC (80\%), whereas $20 \%$ and $40 \%$ of MDSCC cases and $40 \%$ and $30 \%$ of PDSCC cases showed cytoplasmic and combined staining, respectively.

\section{Intensity}

NOM demonstrated predominantly mild staining intensity, whereas the intensity increased progressively from 

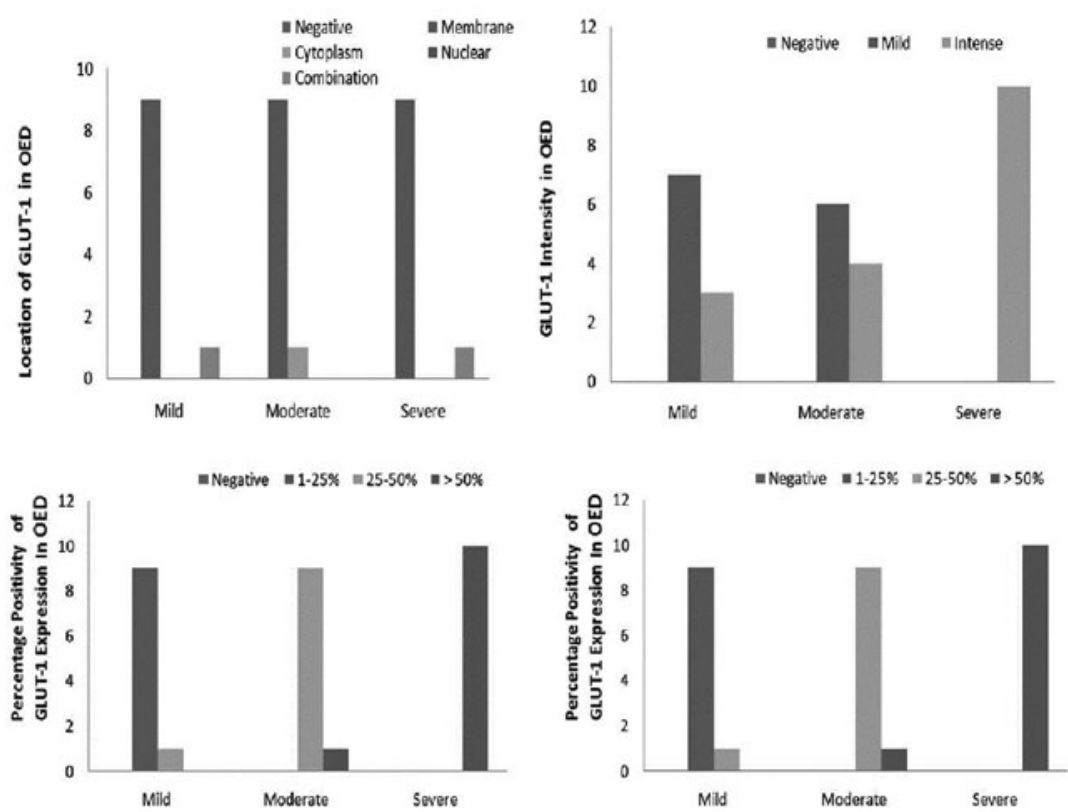

Fig. 3 Variation of GLUT-1 immunoexpression in various grades of OED

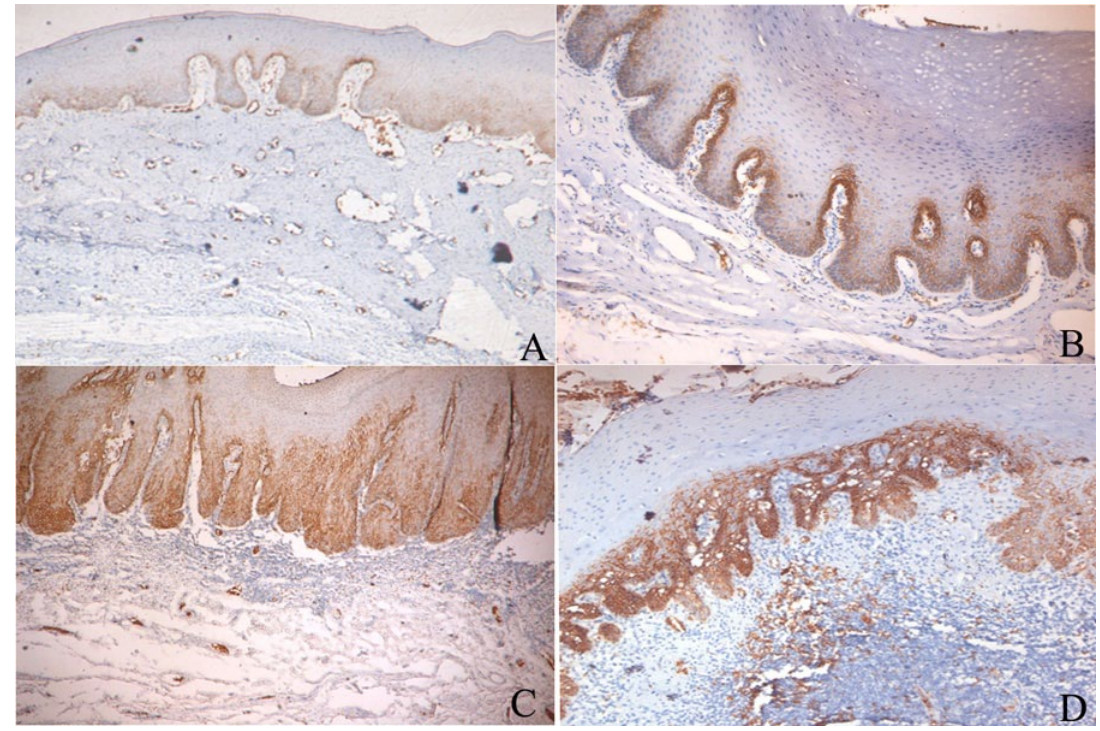

Fig. 4 Mild basal expression of GLUT-1 in normal mucosa $(\mathrm{A}, \times 100)$, whereas intense membrane expression extends to the suprabasal layer in mild OED $(\mathrm{B}, \times 100)$. Intense GLUT-1 expression extends to the spinous layer in moderate OED $(\mathrm{C}, \times 100)$ and to the surface corneal layer in severe $\operatorname{OED}(\mathrm{D}, \times 100)$.

OED to OSCC, and to VC $(P=0.004)$ (Fig. 2). Furthermore, the intensity of staining increased progressively from mild to severe dysplasia $(P=0.009)$. Mild and moderate OED showed mild staining, whereas all cases $(100 \%)$ of severe OED showed intense staining (Figs. 3 , 4). A progressive increase in the intensity of GLUT-1 staining from WDSCC to PDSCC was seen, but this did not reach statistical significance $(P=0.183)$ (Figs. 5, $6)$. VC demonstrated a predominant intense of GLUT-1 expression (Figs. 5, 6).

\section{Percentage}

The percentage of positivity increased progressively from NOM to OED, and to carcinoma, i.e., OSCC and VC $(P<0.001)$. In NOM, the percentage of GLUT-1 expression was usually $1-25 \%$ ( $80 \%$ of samples), but two samples were negative. OED showed positivity in all three ranges, while in OSCC and $\mathrm{VC}$ the range of positivity was predominantly $25-50 \%$ and $>50 \%$ (Fig. 2). A gradual increase of GLUT-1 expression was observed in the OED group as the dysplasia increased from mild to 
severe $(P<0.001)$. Mild OED showed GLUT-1 expression within a range of $0-25 \%$ (90\% of the cases), while moderate OED showed a range of positivity of $25-50 \%$
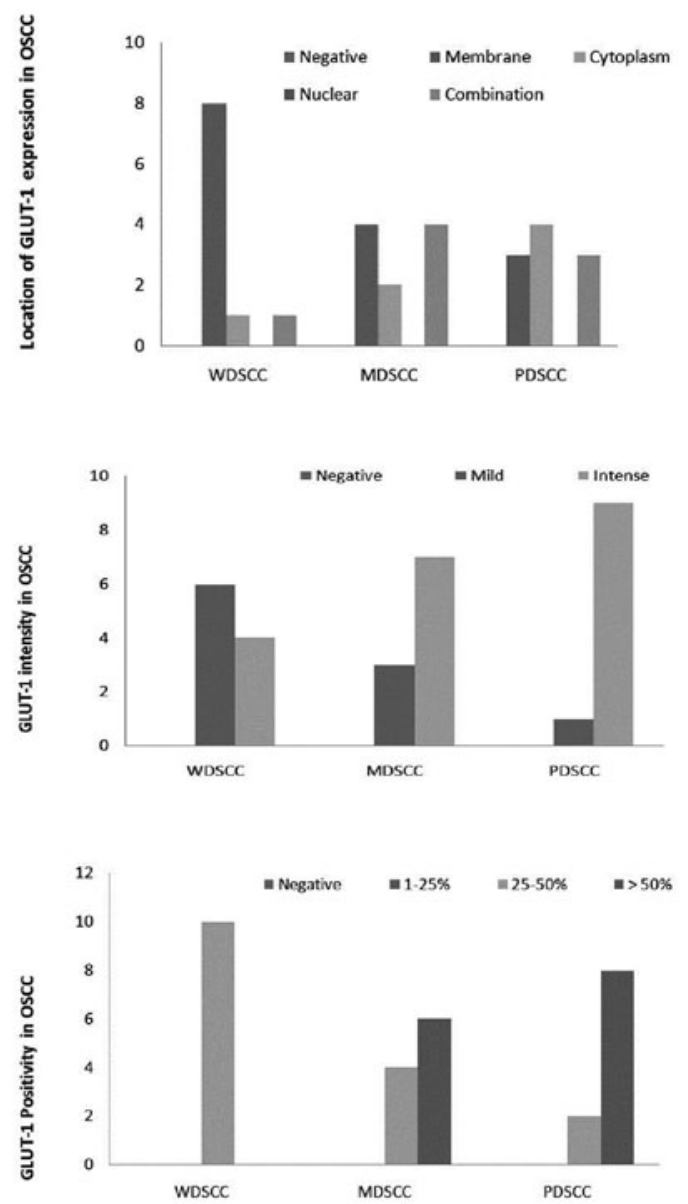

Fig. 5 Various criteria used to evaluate GLUT-1 expression in various grades of OSCC.
(90\%) and severe dysplasia showed $>50 \%$ expression in all cases (100\%) (Figs. 3, 4). A statistically significant increase in the percentage of GLUT-1 expression was also noted in OSCC with progression from WDSCC to $\operatorname{PDSCC}(P=0.015)$ (Figs. 5, 6).

\section{Extent}

The extent of GLUT-1 expression in different strata of the epithelium was evaluated in the OED group. It was limited predominantly to the basal/parabasal layer in mild OED (70\%), increased progressively to the spinous layer in moderate OED (80\%), and extended to the corneal layer in all cases of severe OED (100\%). This difference in the extent of GLUT-1 immunoexpression was statistically significant $(P<0.001)$ (Figs. 3,4$)$.

\section{Discussion}

The metabolism of glucose orchestrates numerous functions, as glucose oxidation serves as the chief source of energy in eukaryotic cells. Consequently, glucose plays a key role in the regulation of transcription, and the activities of hormones, enzymes and neurons. These functions are characteristically dependent on uptake of glucose by cells, which is regulated by the glucose transporter family (GLUT 1-14), whose expression and activity is regulated by oncogenes and growth factors $(6,7)$. Numerous mechanisms and pathways can affect the glycolytic phenotype of both normal and neoplastic cells $(6,7)$. GLUT-1 is the crucial target gene for hypoxia inducible factor (HIF), that mediates glucose influx into cells (7). Thus, upregulation of GLUT-1 is a commonly documented sequel to hypoxia in several cells and tissues.

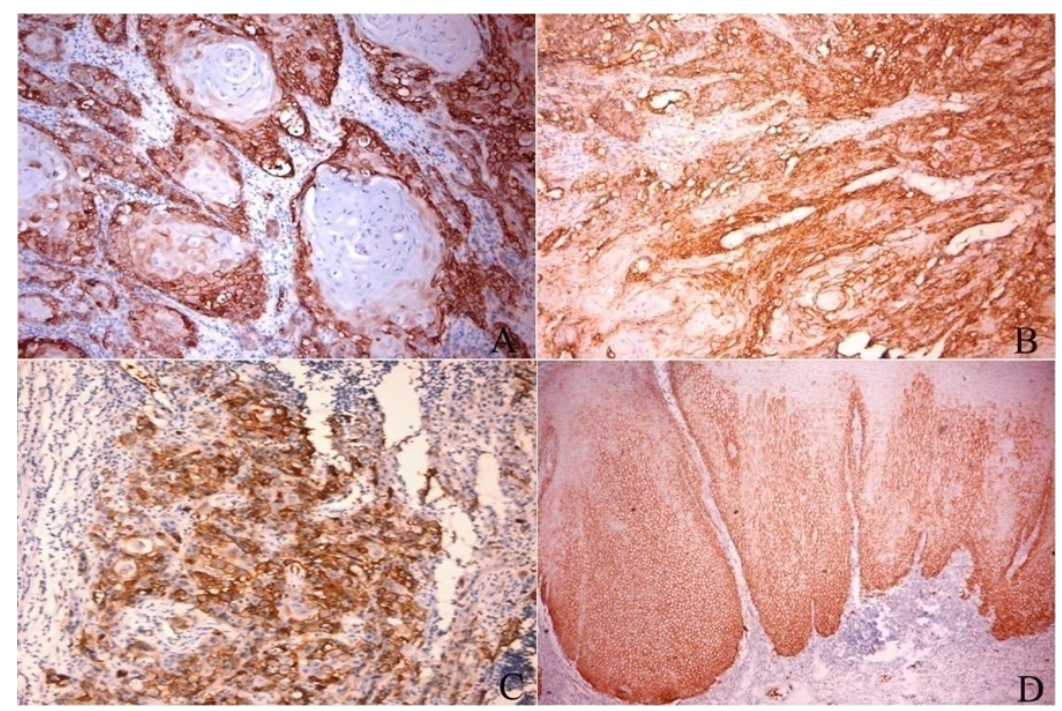

Fig. 6 Intense membranous expression of GLUT 1 in $\operatorname{WDSCC}(\mathrm{A}, \times 100)$. Combined membranous and cytoplasmic GLUT 1 positivity in $\operatorname{MDSCC}(\mathrm{B}, \times 100)$ and PDSCC $(C, \times 100)$. Predominant membrane staining is evident in VC $(\mathrm{D}, \times 100)$. 
GLUT-1 can alter glucose influx under certain conditions that have a higher metabolic requirement, such as cell division (mitosis and meiosis), malignant transformation and nutrient depletion (12). Therefore, deregulated expression of GLUT has been well documented in association with malignancy (6-9). The present study evaluated and compared the expression of GLUT-1 in NOM, OED, OSCC, and VC.

We regarded GLUT-1 expression in NOM (oral epithelium) as the positive control for the present study, weak membrane staining being evident in the basal and suprabasal layers, with no expression evident in the superficial layers. This accords with the findings of Rudlowski et al. and Reisser et al., where the normal mucosa showed mainly membranous staining in the basal layer $(13,14)$. Staining intensity was predominantly weak and showed focal to homogenous variation among the different cell layers. However, intense GLUT-1 positivity was seen in the endothelia of blood vessels in the same tissue samples. These results correspond to the differentiationrelated expression pattern of GLUT-1 in normal human epidermis, which is significant in the basal layer and reduced in suprabasal layer $(14,15)$. The presence of GLUT-1 expression in the basal compartment suggests proliferative activity associated with glucose transport from the basement membrane. With increased maturation, GLUT-1 expression decreases and total absence of GLUT-1 reflects complete maturation and degradation (14). This implies that membrane staining could be an indicator of low proliferative potential, and thus favorable prognosis.

In OED, GLUT-1 expression increased with the severity of dysplasia. In mild dysplasia, GLUT-1 expression was noted in the basal layer and demonstrated predominantly mild staining, whereas in moderate dysplasia, it was intense and extended to the spinous layer. In all cases of severe dysplasia (100\%), the entire epithelium including the granular and corneal layers expressed GLUT-1 strongly. Increased intensity of GLUT-1 staining is reportedly correlated with overexpression of GLUT-1 (16), in accord with several studies of cervical cancer, breast cancer and head and neck carcinoma (7,13-15). This association of GLUT-1 expression with the grade of dysplasia has been previously documented, and has been linked to the glycogen content of the cells, being high in non-dysplastic areas of epithelium and decreased or absent in areas of dysplasia (16). Examination of the glycogen content to establish whether any alterations in glucose uptake are related to tumor-associated changes revealed that glycogen was more abundant in nondividing cells of the superficial layers of benign cervical epithelium. Accordingly, it seems that reduced glycogen levels are significantly associated with increased expression of GLUT-1 as the grade of dysplasia increases $(14,17,18)$.

Furthermore, in the present study, we investigated the percentage of cells that showed GLUT-1 positivity. Two $(16.7 \%)$ of the NOM samples were GLUT-1-negative and $8(80 \%)$ showed between $1-25 \%$ positivity (score 1). In OED, the range of positivity in mild dysplasia was mainly $1-25 \%$ (score 1), whereas nine cases of moderate dysplasia (90\%) showed a positivity range of $26-50 \%$ (score 2) all 10 cases of severe dysplasia (100\%) showed $>50 \%$ positivity (score 3 ). Similar results have been reported for cervical dysplasia by Mendez (15): the number of positive cells increased as the grade increased, reflecting the increased proliferative capacity of the higher-grade lesions $(14,15)$, suggesting that oncogenetriggered mechanisms might be directly involved in GLUT-1 upregulation.

All of the 30 cases of OSCC we investigated were GLUT-1-positive, WDSCC cases usually showing mild staining intensity $(80 \%)$. Expression was predominantly evident at the periphery of well differentiated tumor islands and absent in the central keratin pearls, i.e. a prostromal pattern. In keratin pearls, increased accumulation of glycogen has been reported, correlating inversely with GLUT-1 immunoexpression, suggesting that differentiated and mature cells present in keratinized regions lack GLUT-1 expression (16). The presence of glycogen is known to be related to cellular maturation of squamous epithelium and disappears with loss of differentiation during neoplastic transformation. In the present study, most oral carcinomas demonstrated strong GLUT-1 expression in comparison to NOM and dysplasia. Furthermore, a progressive increase in staining intensity with histologic grade was observed, PDSCC showing the highest intensity $(14,15,19,20)$.

In most of the PDSCC cases, GLUT-1 expression showed an "antistromal" pattern with higher expression in central and perinecrotic zones. This suggests hypoxia-driven GLUT-1 stimulation, which creates an antistromal staining pattern in areas devoid of squamous differentiation/keratinization. Among the $10 \mathrm{MDSCC}$ cases, $3(30 \%)$ showed mild staining intensity and 7 (70\%) showed intense staining. Among the 10 PDSCC cases, only $1(10 \%)$ showed mild staining and $9(90 \%)$ showed intense staining. These findings accord with those of Reisser and Ohba in $\operatorname{OSCC}(8,13)$.

In OSCC, the percentage of cells showing GLUT-1 immunostaining increased with advancing grade, in agreement with previous studies of colorectal and rectal 
carcinomas where the percentage of positivity increased with the carcinoma grade (17-19). GLUT-1 regulates glucose influx into cancer cells, assisting in energy preservation, especially in weakly perfused/hypoxic regions characterized by an inadequate supply of glucose. This explains why GLUT-1 staining is stronger in the central areas tumors and in areas of necrosis located remotely from blood vessels, and lends support to the possibility that hypoxia upregulates GLUT-1. In fact, Reisser et al. have demonstrated GLUT-1 staining in the outer layers of tumor nests, and attributed it to the role of this protein in cellular differentiation, suggesting that it might be able to predict the degree of histologic differentiation (13).

In $\mathrm{VC}$, all of the tissues were positive for GLUT-1 expression (100\%). The expression varied from the suprabasal and superficial layers, with strongly intense staining in $24(80 \%)$ cases and only mild staining in six (20\%) cases (16). Cells at the maturation stage did not express GLUT-1, whereas $13(43.3 \%)$ cases showed an expression range of $26-50 \%$ and $17(56.7 \%)$ one of $>50 \%$. In VC, the majority of cases, i.e. $23(76.7 \%)$, showed membranous staining and 7 (23.3\%) showed combined membranous and cytoplasmic staining. As no other previous study has investigated GLUT-1 expression in VC, it is not possible to compare our results with others.

VC and WDSCC resembled each other in terms of GLUT-1 expression and staining pattern. Predominantly membranous staining was seen in WDSCC $(60 \%)$ and VC (76.6\%). This expression was located mainly at the invasive front of $\mathrm{VC}$, and suggested an increased level of proliferative activity and glucose intake. GLUT-1 expression was lower in central differentiated areas of WDSCC, i.e. a prostromal pattern, which could be inversely related to the glycogen content, as discussed previously $(14,15,19,20)$.

With regard to the location of GLUT-1 expression, anti-GLUT-1 antibody usually recognizes membranebound proteins on epithelial cells. However in our study, GLUT-1 also showed occasional cytoplasmic staining. A range of cellular locations of GLUT-1 expression have been documented in the same tumors, including membranous, cytoplasmic and combined GLUT-1 expression (21). A few studies have suggested that the varying patterns of GLUT-1 expression could have prognostic value, such as the study of Smeland et al. on sarcomas (21). However, some other studies have reached contrary conclusions (22). Most of our present cases showed membranous expression, whereas a few had a combination of both membranous and cytoplasmic staining. NOM showed membrane staining predominantly, while in OED several cases demonstrated combined staining, which appeared to correlate with the transformation of dysplasia to carcinoma. In OSCC also, most of the cases showed membranous expression, although surprisingly MDSCC showed combination staining to a greater degree than PDSCC. GLUT-1 induction following hypoxia involves a succession of changes to its intrinsic activity, kinetics and expression. Initially, there is "unmasking" of the protein that enhances its affinity for glucose. Further stimulation results in translocation of existing glucose transporters from cytoplasmic vesicles to the plasma membrane, and an eventual increase in the synthesis of GLUT-1 mRNA (23). Ariely et al. investigated whether cytoplasmic and membranous expression of GLUT-1 in tumors was related to the duration and extent of hypoxia present in different areas. They suggested that co-localization of GLUT-1 with the Golgi leads to combined membrane and cytoplasmic expression (23).

The present study demonstrated that GLUT-1 has a consistent role in oral premalignant and malignant lesions and that its expression level and activity appear to be associated with malignant transformation and aggressiveness. The expression of GLUT-1 increased with disease severity; OSCC and VC showed the highest intensity, followed by OED and then NOM. The increased GLUT-1 expression associated with the degree of dysplasia reflects the expanding glycolytic response to hypoxia. Furthermore, the upregulation of GLUT-1 with increasing histologic grade in OSCC suggests that in order to proliferate, malignant cells require increased glucose uptake due to their high energy requirement. Our study is the first to have demonstrated prominent GLUT-1 expression in VC, thus indicating its inherent metabolic capacity. These characteristics of GLUT-1 in proliferating cells may be related to the aggressiveness of tumors and their response to various treatment modalities, thus being informative when planning individual treatment approaches.

\section{Acknowledgments}

We acknowledge the statistical assistance of Mr. MD Mallapur and thank Dr. Alka Kale and Dr. Seema Hallikerimath for their support during this study.

\section{References}

1. Jin X, Lu S, Xing X, Wang L, Mu D, He M et al. (2013) Thalidomide: features and potential significance in oral precancerous conditions and oral cancer. J Oral Pathol Med 42, 355-362.

2. Tilakaratne WM, Sherriff M, Morgan PR, Odell EW (2011) Grading oral epithelial dysplasia: analysis of individual 
features. J Oral Pathol Med 40, 533-540.

3. Napier SS, Speight PM (2008) Natural history of potentially malignant oral lesions and conditions: an overview of the literature. J Oral Pathol Med 37, 1-10.

4. van der Waal I (2009) Potentially malignant disorders of the oral and oropharyngeal mucosa; terminology, classification and present concepts of management. Oral Oncol 45, 317-323.

5. Chaudhary M, Gadbail AR, Vidhale G, Mankar Gadbail MP, Gondivkar SM, Gawande M et al. (2012) Comparison of myofibroblasts expression in oral squamous cell carcinoma, verrucous carcinoma, high risk epithelial dysplasia, low risk epithelial dysplasia and normal oral mucosa. Head Neck Pathol 6, 305-313.

6. Carvalho KC, Cunha IW, Rocha RM, Ayala FR, Cajaíba MM, Begnami MD et al. (2011) GLUT1 expression in malignant tumors and its use as an immunodiagnostic marker. Clinics (Sao Paulo) 66, 965-972.

7. Kunkel M, Reichert TE, Benz P, Lehr HA, Jeong JH, Wieand $S$ et al. (2003) Overexpression of Glut-1 and increased glucose metabolism in tumors are associated with a poor prognosis in patients with oral squamous cell carcinoma. Cancer 97, 1015-1024.

8. Ohba S, Fujii H, Ito S, Fujimaki M, Matsumoto F, Furukawa $\mathrm{M}$ et al. (2010) Overexpression of GLUT-1 in the invasion front is associated with depth of oral squamous cell carcinoma and prognosis. J Oral Pathol Med 39, 74-78.

9. Eckert AW, Lautner MH, Schütze A, Taubert H, Schubert J, Bilkenroth U (2011) Coexpression of hypoxia-inducible factor- $1 \alpha$ and glucose transporter- 1 is associated with poor prognosis in oral squamous cell carcinoma patients. Histopathology 58, 1136-1147.

10. Barnes L, Eveson JW, Reichart P, Sidransky D (2005) WHO classification of tumours. Pathology and genetics of head and neck tumours. IARC Press, Lyon, 177-179.

11. Rajendran R, Sivapathasundaram B (2009) Shafer's textbook of oral pathology. 6th ed, Elsevier, 106-107.

12. Jonathan RA, Wijffels KI, Peeters W, de Wilde PC, Marres HA, Merkx MA et al. (2006) The prognostic value of endogenous hypoxia-related markers for head and neck squamous cell carcinomas treated with ARCON. Radiother Oncol 79, 288-297.

13. Reisser C, Eichhorn K, Herold-Mende C, Born AI, Bannasch
P (1999) Expression of facilitative glucose transport proteins during development of squamous cell carcinomas of the head and neck. Int J Cancer 80, 194-198.

14. Rudlowski C, Becker AJ, Schroder W, Rath W, Büttner R, Moser M (2003) GLUT1 messenger RNA and protein induction relates to the malignant transformation of cervical cancer. Am J Clin Pathol 120, 691-698.

15. Mendez LE, Manci N, Cantuaria G, Gomez-Marin O, Penalver M, Braunschweiger P et al. (2002) Expression of glucose transporter-1 in cervical cancer and its precursors. Gynecol Oncol 86, 138-143.

16. Ayala FR, Rocha RM, Carvalho KC, Carvalho AL, da Cunha IW, Lourenço SV et al. (2010) GLUT1 and GLUT3 as potential prognostic markers for oral squamous cell carcinoma. Molecules 15, 2374-2387.

17. Cooper R, Sarioğlu S, Sökmen S, Füzün M, Küpelioğlu A, Valentine $\mathrm{H}$ et al. (2003) Glucose transporter-1 (GLUT-1): a potential marker of prognosis in rectal carcinoma? $\mathrm{Br} \mathrm{J}$ Cancer 89, 870-876.

18. Kim CH, Kim MY (2012) Correlation between glucose transporter type-1 expression and ${ }^{18} \mathrm{~F}-\mathrm{FDG}$ uptake on PET in oral cancer. J Korean Assoc Oral Maxillofac Surg 38, 212-220.

19. Haber RS, Rathan A, Weiser KR, Pritsker A, Itzkowitz SH, Bodian C et al. (1998) GLUT1 glucose transporter expression in colorectal carcinoma: a marker for poor prognosis. Cancer 83, 34-40.

20. Brown RS, Goodman TM, Zasadny KR, Greenson JK, Wahl RL (2002) Expression of hexokinase II and Glut-1 in untreated human breast cancer. Nucl Med Biol 29, 443-453.

21. Smeland E, Kilvaer TK, Sorbye S, Valkov A, Andersen S, Bremnes RM et al. (2012) Prognostic impacts of hypoxic markers in soft tissue sarcoma. Sarcoma 2012, 541650.

22. Kunkel M, Moergel M, Stockinger M, Jeong JH, Fritz G, Lehr HA et al. (2007) Overexpression of GLUT-1 is associated with resistance to radiotherapy and adverse prognosis in squamous cell carcinoma of the oral cavity. Oral Oncol 43, 796-803.

23. Airley R, Loncaster J, Davidson S, Bromley M, Roberts S, Patterson A et al. (2001) Glucose transporter Glut-1 expression correlates with tumor hypoxia and predicts metastasis-free survival in advanced carcinoma of the cervix. Clin Cancer Res 7, 928-934. 